\title{
Correction to: Two-year follow-up of one-stage left unilateral thoracoscopic epicardial and transcatheter endocardial ablation for persistent and long-standing persistent atrial fibrillation
}

\begin{abstract}
Carlo de Asmundis ${ }^{1} \cdot$ Varnavas Varnavas $^{1} \cdot$ Juan Sieira $^{1} \cdot$ Erwin Ströker $^{1} \cdot$ Henrique E. Coutiño ${ }^{1} \cdot$ Muryo Terasawa $^{1}$. Juan Pablo Abugattas ${ }^{1}$ • Francesca Salghetti $^{1}$ - Riccardo Maj ${ }^{1}$. Osório Thiago Guimarães ${ }^{1}$ • Saverio lacopino ${ }^{1}$. Vincent Umbrain ${ }^{2} \cdot$ Jan Poelaert $^{2} \cdot$ Pedro Brugada $^{1} \cdot$ Sandro Gelsomino ${ }^{3} \cdot$ Gian-Battista Chierchia ${ }^{1} \cdot$ Mark La Meir $^{4}$
\end{abstract}

Published online: 17 January 2020

(C) Springer Science+Business Media, LLC, part of Springer Nature 2020

\section{Correction to: J Interv Card Electrophysiol.} https://doi.org/10.1007/s10840-019-00616-w

Owing to a type error during final correction and proof data submission to the journal, there were mistakes introduced into the legends of Fig. 3 and Fig. 4 as well as into the Y-axis title of the plot of Fig. 4.

The legend of Figs. 3 and 4 and the Y-axis title of the plot in Fig. 4 should read as follows.

Fig. 3 Endocardial ablation. (a) Three dimensional (3-D) reconstruction of the left atrium (LA) and of the pulmonary veins (PVs) based on cardiac computer tomography (CT); (b) 3-D voltage mapping of the LA and PVs demonstrating antral isolation of the PVs, but not of the posterior wall (PW).

Fig. 4 Kaplan-Meier curve for freedom from AF/AT. AF indicates atrial fibrillation; AT, atrial tachycardia; PerAF, persistent atrial fibrillation; LS-PerAF, long-standing persistent atrial fibrillation

Fig. 4 (Plot) Y-axis title: AF / AT Freedom

The authors apologize for these errors.

The online version of the original article can be found at https://doi.org/ 10.1007/s10840-019-00616-w

Carlo de Asmundis

carlo.deasmundis@uzbrussel.be; carlodeasmundis@me.com

1 Heart Rhythm Management Centre, Postgraduate course in Cardiac Electrophysiology and Pacing, Vrije Universiteit Brussel,

Universitair Ziekenhuis Brussel, Laarbeeklaan 101,

1090 Brussels, Belgium

2 Anesthesiology Departement, Vrije Universiteit Brussel, Universitair Ziekenhuis Brussel, Brussels, Belgium

3 Department of Heart and Vessels, Careggi Hospital, Florence, Italy

4 Cardiac Surgery Department, Vrije Universiteit Brussel, Universitair Ziekenhuis Brussel, Brussels, Belgium 


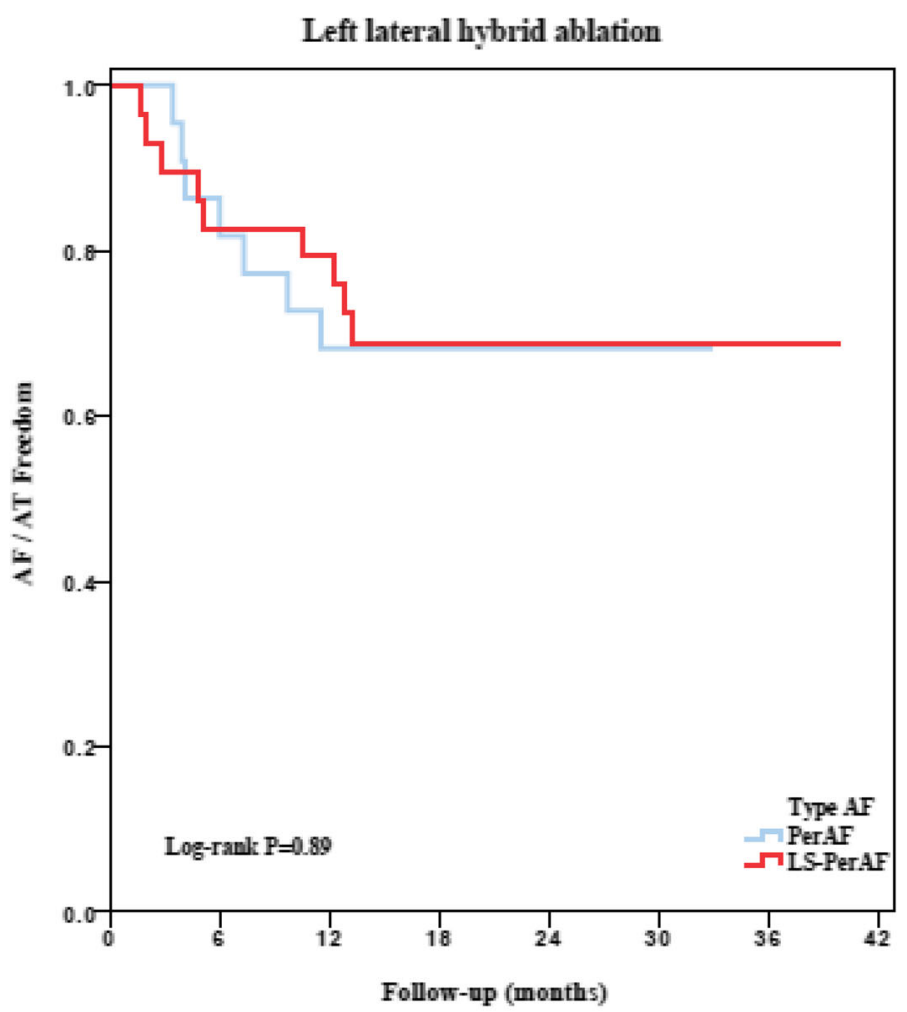

Pafients at risk:

$\begin{array}{lllllllll}\text { PerAF } & 22 & 19 & 15 & 15 & 15 & 15 & 15 & 15\end{array}$

LS-PerAF

$29 \quad 25 \quad 24$

20

20 